\title{
Competencias lecto-escritoras en educación media: Deconstrucción, Reconstrucción y Evaluación de la práctica Pedagógica
}

\section{Reading and writing skills in secondary education: Deconstruction, Reconstruction and Evaluation of pedagogical practice}

\section{Lisset Vargas Minorta ${ }^{1}$}

\section{Audin Aloiso Gamboa Suárez ${ }^{2}$}

\section{Raúl Prada Núñez ${ }^{3}$}

\section{RESUMEN}

El presente artículo de investigación muestra los resultados de un estudio que prentendió comprender el desarrollo de competencias lecto-escritoras desde la deconstrucción, reconstrucción y evaluación de la práctica

1 Docente Escuela Normal Superior María Auxiliadora (Cúcuta - Norte de Santander), Magíster en Práctica Pedagógica (Universidad Francisco de Paula Santander (Cúcuta - Colombia), correo electrónico: Ivargs@gmail. com, Orcid: orcid.org/0000-0001-6001-5720.

2 Docente Universidad Francisco de Paula Santander (Colombia), Doctor en Ciencia de la Educación (Universidad de Cartagena - Colombia), correo electrónico: audingamboa@ufps.edu.co, Orcid: 000-0001-975-6408.

3 Docente Universidad Francisco de Paula Santander (Colombia), Magíster en Ingeniería de Análisis de Datos, Mejora de Procesos y Toma de Decisiones (Universidad Politécnica de Valencia - España), correo electrónico: raulprada@ufps.edu.co, Orcid: 0000-0001-6145-1786. pedagógica de actores educativos en una institución educativa de básica y media en Colombia. El contexto metodológico se centra en la investigación acción pedagógica. Las técnicas de recolección de la información utilizadas en el estudio fueron los grupos focales y la observación participante. Se concluye que la práctica pedagógica para el desarrollo de competencias lecto-escritoras, mediante el fortalecimiento de la literacidad creativa, es interpretada como una alternativa pedagógica en el aula, la cual posibilita nuevas prácticas y espacios letrados en la institución. 


\section{PALABRAS CLAVE}

Competencias; investigación acción pedagógica; lecto-escritura; práctica pedagógica.

\section{ABSTRACT}

This research article shows the results of a study that sought to understand the development of reading-writing skills from the deconstruction, reconstruction and evaluation of the pedagogical practice of educational actors in a basic and high school educational institution in Colombia. The methodological context focuses on pedagogical action research. The information gathering techniques used in the study were focus groups and participant observation. It is concluded that the pedagogical practice for the development of reading-writing skills, through the strengthening of creative literacy, is interpreted as a pedagogical alternative in the classroom, which enables new practices and literate spaces in the institution.

\section{KEYWORDS:}

Competencies; pedagogical action research; reading-writing; pedagogical practice

\section{INTRODUCCIÓN}

El concepto de competencia generalmente es entendido como el conocimiento que se posee y el uso que se hace de ese conocimiento para resolver una tarea en una situación específica de acuerdo con unas necesidades y exigencias concretas (Gamboa, 2016). La competencia es una categoría pensada desde la formación de los sujetos, en diferentes dimensiones de su desarrollo, y surge como una fina y delicada combinación de componentes diversos, que se pone en escena con regularidad frente a diversas tareas y que, en consecuencia, se manifiesta a través de la acción misma. Es claro entonces que el nivel de desarrollo de competencia solo se visualiza a través de desempeños, de acciones, sea en el campo social, cognitivo, ético, cultural, estético o físico.

El mundo actual experimenta una revolución a partir de los continuos avances tecnológicos, haciendo incierto el futuro y generando cambios en el estilo de vida de las nuevas generaciones; frente a este hecho, la posibilidad del ser humano es sacar lo mejor de sí mismo para lograr flexibilidad y productividad (Robinson, 2011). En este sentido, los retos planteados por la sociedad de la información y del conocimiento, requieren de una mirada hacia los saberes que circulan en la escuela. Por tanto, el desarrollo de la creatividad a partir de prácticas de literacidad abre una nueva perspectiva a la enseñanza de las primeras letras que emergió junto con la escuela en el siglo XIX, posibilitando repensar el concepto de creatividad y enseñanza del lenguaje.

A partir del contexto descrito anteriormente, la educación de hoy exige una formación por competencias, pues los jóvenes de hoy enfrentarán retos aún desconocidos; esto implica una formación que desarrolle las habilidades necesarias para aprender en cualquier momento de la vida, para trabajar en equipo, para comunicarse asertivamente y para resolver los problemas que se presenten en cualquier momento de la vida. De esta manera, en términos de Zubiría (2013), las competencias se entienden como aprehendizajes integrales que se traducen en un saber hacer, un saber sentir y un saber pensar.

De este modo, en el presente trabajo de investigación se hace necesario plantear a partir del enfoque de formación por competencias, el concepto de creatividad como un pensamiento enseñable en el ser humano. De Bono (2004), propone el concepto de pensamiento lateral para hablar de pensamiento creativo, desarrollado a través de la técnica de los seis sombreros, explica la manera de discutir e intercambiar ideas en grupo y concluye que el pensamiento 
creativo puede ser enseñado, pues se requiere un desplazamiento lateral para buscar otros enfoques, otras alternativas.

En este mismo sentido, Robinson (2011) y Hernández, Suárez y Gamboa (2017) afirman que la creatividad se aprende del mismo modo en que se aprende a leer o las matemáticas; por tanto, expresa que la enseñanza en sí es un proceso creativo. Así que, las escuelas que buscan educar las generaciones del siglo XXI, requieren reconfigurar la enseñanza, pues ante la incertidumbre del futuro los niños y jóvenes necesitan una educación que los prepare para los desafíos, puesto que, para este autor la vida es un proceso creativo, que se puede recrear. Por esta razón, la creatividad es necesaria en un mundo que requiere del desarrollo de competencias en el ser humano.

De esta manera, el pensamiento lateral puede usarse en dos sentidos: "específico: una serie de técnicas sistemáticas que se usan para cambiar los conceptos y percepciones y generar otros nuevos. General: exploración de múltiples posibilidades y enfoques, en vez de aceptar un punto de vista único" (De Bono, 2004:98). En este sentido, propone una relación entre los procesos de pensamiento con el color de seis sombreros, asociando los colores de la siguiente manera: blanco con lo observado de forma objetiva, rojo con las sensaciones y sentimientos, negro con las críticas negativas de lo observado, amarillo con las ventajas y aspectos positivos de la observación, verde con las posibles alternativas de solución ante las dificultades observadas y azul con la capacidad de síntesis.

Lo anterior, posibilita la enseñanza del pensamiento creativo o pensamiento lateral, entendido desde un enfoque sociocultural como literacidad crítica, para reconocer que el discurso también tiene ideología. En este sentido, comprender implica la reconstrucción del contenido, de la ideología para darle sentido desde el mundo individual del lector (Cassany, 2006; Moreira y Moreira, 2020; Rivas, 2020).

Desde la noción de pensamiento lateral, la criticidad es comprendida como la posibilidad de leer la ideología que se halla en los discursos que circulan en determinados grupos sociales, los cuales definen sus características e intencionalidades teniendo en cuenta las relaciones de poder, así se entiende por lectura crítica el desarrollo de consciencia del lector para que valore las representaciones y distribuciones de poder que se encuentran de forma implícita en la cultura de lo escrito (Cassany, 2006).

Desde el planteamiento de los nuevos estudios de literacidad y del desarrollo del pensamiento creativo, las prácticas letradas contemporáneas implican la lectura multimodal, al contrario de las tradicionales, que usan otros modos para representar y transmitir información, de modo que:

integran diferentes modos comunicativos junto a la letra. Sin duda la lectura de textos multimodales es más compleja porque el significado no procede sólo de lo que aporta cada modo por separado, sino de la interacción que se produce en el conjunto (Cassany (2008:70).

Por su parte, Henao y Ramírez (2007) evidencia las diferencias en la forma como los lectores exploran y construyen significados en un texto hipermedial, con el propósito de orientar a los docentes para la utilización eficiente y creativa de los recursos hipermediales para mejorar el aprendizaje y la comprensión lectora. Así mismo, Levratto y Aparici (2014) reconocen que la alfabetización digital no es una simple imprenta del nuevo siglo, sino una nueva cultura, por consiguiente los códigos semióticos hacen parte de la cotidianidad, del estilo de vida; su lectura requiere de: reflexión crítica, acción, transición de medios, humanismo y creatividad. 
Del mismo modo, para Jurado, Bustamante y Pérez (1998) la hipertextualidad abarca los diversos formatos de lectura: narrativa icónica, los tipos de interacción oral y escrita y los saberes cotidianos. Diversidad que requiere de nuevos modos de leer y escribir. Por tanto, la lectura crítica busca el reconocimiento de los usos sociales de los nuevos formatos de lectura y escritura.

Con un sentido amplio, Lomas (2015) resignifica el rol del maestro ante la cultura hipermedial, pues es claro que las formas de acceder a la información han cambiado antes los nuevos formatos de lectura y escritura, las cuales tienen un soporte multimedial. Situación que plantea el reto de la alfabetización digital para acceder a la cultura de la imagen, integrada con la palabra y el sonido (Monteiro y Leite, 2021; De la Torre y Mora, 2021).

Por las razones expuestas, la labor del maestro en este siglo debe trascender, generando impacto y nuevas formas de concebir el proceso de enseñanza- aprendizaje. No basta con transmitir cultura, se requiere el desarrollo de competencias para abordar la información diversa y amplia que circula en el mundo, con el propósito de ofrecer alternativas de solución a los retos que plantea la cotidianidad del silgo XXI. Por tanto, expresado por Zuluaga (1999) el maestro requiere reconfigurar su rol, en medio de las tensiones que posibilitan la escuela, puede pensarse y actuar como un intelectual y no como un simple ejecutor de técnicas.

\section{ASPECTOS METODOLÓGICOS}

En la presente investigación se propuso el enfoque cualitativo con el fin de profundizar en la comprensión de la práctica pedagógica para el desarrollo de competencias comunicativas mediante el fortalecimiento de la literacidad creativa de quienes actúan e interactúan en los escenarios escolares. En este sentido, las fases de deconstrucción de la práctica, construcción de la propuesta, implementación de la misma y evaluación de su efectividad, fueron apoyadas en los métodos etnográficos (Martínez, 2004), hermenéuticos y desde la complementariedad.

La información hallada en cada una de las fases se trianguló a través de los instrumentos implementados y de los informantes seleccionados para ello; entendiendo a partir de Martínez (2004), que la categorización de la información hallada permitió la comprensión del sentido que le dieron los estudiantes como actores al problema abordado. De acuerdo con la triangulación de los informantes y de los instrumentos, emergieron categorías que permitieron establecer tres categorías axiales: prácticas letradas instruccionales, formación por competencias desde la literacidad, aprendizaje del lenguaje en las prácticas creativas de literacidad y trabajo colaborativo (Henao y Ramírez, 2010).

los participantes de la investigación fueron 25 jóvenes de décimo grado de la media técnica del Instituto Técnico Industrial Salesiano (Cúcuta Colombia), quienes contaron con la autorización de sus acudientes para participar en el proyecto y el par académico fue el jefe de la Dimensión Comunicativa y una docente investigadora. Para la selección de los informantes se establecieron los siguientes criterios: interés por la lectura y escritura, utilización de las TICs, estudiantes con dificultades en el área de lengua castellana y disponibilidad para participar en la investigación.

\section{RESULTADOS Y DISCUSIÓN}

La investigación se desarrolló en tres fases: 1). Deconstrucción: En esta fase de deconstrucción de los actuales procesos de literacidad en las prácticas pedagógicas, se buscó la preconfiguración de la realidad al lograr lo expresado por Murcia y Jaramillo (2008:100) como: un acercamiento muy superficial con el fenómeno de estudio, la primera aproximación refleja una pre-estructura; la que al ser 
interpretada, muestra una esencia inicial de la realidad estudiada. 2. Reconstrucción: Esta fase consistesegúnMejía(2004)generalaoportunidad de construir, de experimentar colectivamente una nueva mirada de lo pedagógico. 3. Evaluación: evalúa desde la realidad misma con las categorías preestablecidas, buscando una lectura crítica, es decir de la red de relaciones que posibilitaron la configuración de los relatos y descripciones naturales, la reflexión sobre esos hechos o rasgos culturales, las percepciones que el investigador se ha formado, y las confrontaciones con la teoría formal, deben ser la base de la interpretaciones.

\section{DECONSTRUCCIÓN DE LA PRÁCTICA PEDAGÓGICA DESDE LAS COMPETENCIAS LECTOESCRITORAS}

En cuanto a la formación por competencias, los estudiantes consideran que tanto en el contexto familiar como en el escolar no son estimulados para el desarrollo de habilidades comunicativas, que le permitan un saber, para ser y saber hacer en un contexto determinado; esto se expresa como una de las causas de la falta de comprensión crítica de lo leído, cuando asumen que: "hay poca estimulación a la lectura desde la familia, desde pequeños no nos enseñaron el hábito de la lectura" (Arias, 2018).

Ahora bien, desde el escenario escolar, el maestro vivencia la tensión entre lo establecido en los lineamientos y estándares dados por el Ministerio de Educación Nacional (MinEducación, 2006) y la necesidad de partir de los intereses y necesidades del entorno de los estudiantes, ya que se cree que si no se abordan los temas no se desarrollan las habilidades y competencias del área de lenguaje. Por tanto la maestra manifiesta en las bitácoras que: "es necesario articular los procesos de producción escrita con el desarrollo de habilidades de la oralidad y desarrollar habilidades en los estudiantes para abordar los diversos géneros de producción de texto oral" (Vargas, 2018)

\section{RECONSTRUCCIÓN DE LA PRÁCTICA PEDAGÓGICA DESDE LAS COMPETENCIAS LECTOESCRITORAS}

Los retos de la educación actual plantean la formación por competencias como una opción curricular. En el ámbito comunicativo, la lectura y la escritura trascienden la escuela, aunque las prácticas letradas escolares resultan poco interesantes para los jóvenes de hoy. Por tal razón, la intervención de la presente investigación se centró en el fortalecimiento de la competencia comunicativa- escritora (Derechos básicos de aprendizaje, lenguaje); generando una dinámica de participación activa de los estudiantes, quienes se reconocen como usuarios de los lenguajes del mundo contemporáneo. De este modo, los estudiantes frente a la propuesta implementada, expresan que "este acto fue todo un proceso, fue y es cosas, cosas que nos llenan para la vida y nos servirán muchísimo a futuro" (Moncada, 2018). Así mismo, señalan que "personalmente en el aspecto de escritura, en un principio se me hizo complicado, no entendía unas cosas y en el momento de redactar me confundía en otras" (Torres, 2016)

Según lo planteado en las bitácoras, los estudiantes comprendieron la escritura como un proceso que requiere de múltiples revisiones, de reescribir y cuestionar lo que se plantea; en los instrumentos se encuentran expresiones que evidencian lo anterior:

Así, en el grupo de apoyo al proceso de escritura en el grado décimo, se revisaron los escritos iniciales a partir de las rúbricas, haciendo sugerencias por parte de los pares para mejorarlos. Además, se revisó el estilo de escritura de acuerdo 
con lectores a quienes se proyecta. La planeación de la escritura les permitió a los estudiantes comprender que no es un ejercicio único, sino que es resultado de todo un proceso en el que pueden intervenir diversas personas (Bitácora 1).

Se entregaron los escritos a los estudiantes de cada uno de los cursos para que durante la misma clase hicieran la reescritura del texto según las sugerencias de los compañeros y la maestra. (Bitácora 10).

En contraste con lo registrado en las bitácoras, en las narrativas testimoniales los estudiantes expresan algunas percepciones y fortalezas con la implementación del proceso de escritura:

"esto te ayuda a que mejores el léxico y es muy útil para la vida. Fue una experiencia satisfactoria, ya que todo esto es necesario e importante no sólo ahora, siempre lo será" (Hernández, 2018)

También, en la estrategia fue necesario buscar el desarrollo de la competencia comunicativalectora, orientando el proceso de lectura según los momentos planteados por Solé (1998). Lo anterior se reflejó en la las bitácoras de la siguiente manera:

La actividad de escritura que se iniciaba, requería recordar a los estudiantes las pautas según los momentos de lectura que establece Isabel Solé (1998): antes de la lectura, durante la lectura y después de la lectura; la maestra explicó cada uno de los momentos (Bitácora 8).

Se revisó el estilo de escritura de acuerdo con lectores a quienes se proyecta. En cuanto a la competencia comunicativaescritora, las actividades diseñadas en la propuesta, buscaron el fortalecimiento de los componentes de dicha competencia: semántico, sintáctico y pragmático. De esta manera, en la implementación se creó la necesidad de aprender los elementos del leguaje escrito en los estudiantes, algunas expresiones de las bitácoras y en la narrativa testimonial de uno de los estudiantes (Bitácora 3).

La maestra orientó a los estudiantes para la elaboración del escrito. Para ello, se organizaron pequeños equipos (máximo tres estudiantes) y se explicó nuevamente la estructura básica de todo texto periodístico y argumentativo (Bitácora 1).

En los equipos de trabajo los estudiantes seleccionaron el tipo de escrito que elaborarían; también hicieron una lluvia de ideas de los temas sobre los cuales buscarían los referentes necesarios para la elaboración del escrito. Fue necesario orientar la lectura de insumos de ortografía, argumentación y de redacción. Además, se revisó el estilo de escritura de acuerdo con lectores a quienes se proyecta (Bitácora 10).

\section{EVALUACIÓN DE LA PRÁCTICA PEDAGÓGICA DESDE LAS COMPETENCIAS LECTOESCRITORAS}

Un enfoque curricular por competencias, impacta los propósitos de los aprendizajes que circulan en la escuela. Además, en la actualidad la dificultad ante el conocimiento no es la escasez de información, sino la saturación, accediendo a través de una ventana electrónica del televisor o del ordenador (Lomas, 2015). Por tal razón, el rol del maestro se reconfigura en el acompañamiento a las formas como los estudiantes buscan y seleccionan la información en la web. En la intervención, los estudiantes expresan:

pues más que todo lo que implementamos fue en las lecturas ya que no necesariamente 
teníamos que tener el libro, sino que lo podíamos descargar por pdf en el celular y la comunicación con la profesora por diferentes grupos sociales o Whatsapp (García, 2018).

Así mismo aseveran que "en lectura se nos enseñó cómo buscar artículos académicos, cómo referenciarlos y cómo utilizar la información que encontramos en ellos para poder sustentar un escrito" (Suárez, 2018).

Desde el anterior enfoque de lectura y escritura, las competencias comunicativas abarcan diversos elementos lingüísticos necesarios para la comprensión por parte del lector; así, las práctica letradas escolares permitieron que los estudiantes se acercaran a estos saberes con una dinámica en la que el texto fue percibido como un dispositivo de comunicación (Jurado y Bustamante, 2015). La anterior concepción del texto, permitió generar una dinámica de interacción entre los escritores y lectores, roles que se invertían para asumir el compromiso de leer y escribir entre pares (Carlino, 2013). Los estudiantes lo percibieron, manifestando que: "pues, me parece muy bien, ya que no entregamos un texto final sino que fue un proceso pues que cada vez se iba construyendo, mejorándolo y poniendo puntos a favor y corrigiendo las cosas que estaban mal" (Pérez, 2018).

A partir de los componentes de la competencia comunicativa escritora se buscó el desarrollo de esta, abordando el proceso de revisión de escritura entre pares (Carlino, 2013). Para ello, se diseñó a partir de los aprendizajes de cada componente (semántico, sintáctico, pragmático) una rúbrica de revisión de los escritos. En las bitácoras la maestra deja registro de ello: Se hizo retroalimentación de los criterios establecidos en cada una de las parejas, al final se elaboraron unos criterios generales a partir de lo propuesto por cada pareja (Bitácora 4).
Fue importante, tener en cuenta el análisis de las pruebas saber institucionales (2017), en las cuales se presentan las debilidades y las fortalezas en cada de los componentes de las dos competencias comunicativas que se evalúan: componente semántico, sintáctico y pragmático. En este sentido, el componente sintáctico, es señalado como una debilidad en los estudiantes que actualmente cursan el grado décimo. Por tal motivo, una de las finalidades de la intervención fue el desarrollo de dicho componente. Los estudiantes expresan que en la competencia comunicativa escritora lograron fortalecer aprendizajes como: "en la escritura me ayudó mucho con la ortografía y el orden" (Ramírez, 2018), "en la escritura mejoramos la redacción, antes eran frases simples, frases que no tenían estructura, luego fuimos implementando los conectores, los usos de sinónimos, nosotros nos quedamos en las mismas palabras y pues ya ampliamos" (Hernández, 2018). Así mismo, los estudiantes afirman que "se requiere a la hora de saber escribir, la coherencia, cohesión en ese momento y reglas ortográficas" (Mendez, 2018).

Igualmente, otro de los aprendizajes que se generaron en los diversos cursos del grado décimo relacionado con el saber ser en un contexto determinado, evidencia que los aprendizajes permitieron el desarrollo de la competencia en todos sus desempeños: aprender a conocer, a hacer, a ser y a convivir en un contexto específico (Zubiría, 2013). Uno de los estudiantes afirma que: "pues aprendí, a valorar o calificar un escrito teniendo en cuenta diferentes aspectos como puede ser la coherencia, el léxico, o sea a valorar un escrito... digamos, desglosar un escrito, desglosar sus características y de esa forma dar una valoración mucho más detallada. Aprendí a valorar un escrito de esa manera" (Caballero, 2018). También aceveran que "en el aspecto de corrección, no fue muy complicado, además la rúbrica fue una ayuda esencial porque tenía 
todos los componentes muy claros, también las explicaciones y ayudas dadas por la docente fueron sustanciales" (Becerra, 2018).

También para los estudiantes del grupo líder de la propuesta, la escritura permitió fortalecer la competencia comunicativa- lectora, estableciendo una relación entre la lectura y la escritura; uno de los registros del grupo evidencian lo anterior de la siguiente manera:

primero que todo con la lectura fue algo un poco nuevo, porque leía solamente cosas de mi gusto. Entonces este año me enfrenté a un vocabulario más complejo y el léxico realmente me dio un poco duro. Creo que son pensamientos muy chéveres, me pareció más que todo chévere conocer cómo pensaban antes compadado con lo que pensamos ahora. Aprendí cómo analizar, valorar y a ver lo positivo a todas las interpretaciones que hubo y pensar en cómo y por qué pensaba así. Creo que eso fue lo que más aprendí de la lectura, de la escritura me encantó poder comentar, porque uno siempre que lee algo lo piensa y lo piensa para uno, pero realmente uno como que no le pone mucha atención; cuando realmente uno lo escribe, uno se da cuenta de muchas otras cosas; entonces creo que eso fue lo más chévere, $y$, aprendí a leer, aprendí a interpretar, aprendí a analizar, aprendí a comentar (Serrano, 2018).

\section{CONCLUSIONES}

En las estrategias implementadas para el desarrollo de la competencia lecto-escritora se logró la resignificación del rol del maestro y los estudiantes, en el que se dan otros sentidos a los sujetos que hacen parte de la situación didáctica- comunicativa. Sin embargo, es importante reconocer que para los estudiantes es necesaria la instrucción en los saberes que constituyen la competencia escritora que se buscó fortalecer por parte de la maestra, quien asume el acompañamiento de dichos procesos.
La anterior situación didáctica de la enseñanza del lenguaje $\mathrm{y}$ el fortalecimiento de las competencias comunicativas, es un referente que marca posibilidades pedagógicas y didácticas en la institución objeto de estudio, asumiendo la tarea de formación basada en competencias desde una visión crítica y sociocultural de las prácticas letradas contemporáneas, que reconocen al sujeto alfabetizado como un productor de sentidos en los discursos que saturan los diversos dominios letrados (familia, escuela, ocio, hogar y TICs).

Por otra parte, la práctica pedagógica para el desarrollo de competencias comunicativas, mediante el fortalecimiento de la literacidad creativa es interpretada como una alternativa de pedagógica en el aula escolar, la cual posibilitó en la escuela nuevas prácticas y espacios letrados, generando una dinámica de participación activa de los estudiantes al abordar la creatividad desde la criticidad, haciendo mayor énfasis en la implementación de estrategias pedagógicas y didácticas para fortalecer el componente sintáctico de la competencia escritora y proponiendo alternativas de solución a los problemas observados en el contexto institucional.

\section{REFERENCIAS BIBLIOGRÁFICAS}

Carlino, P. (2013). Alfabetización académica diez años después. Revista Mexicana de Investigación Educativa, 18 (57), 355-381.

Cassany, D. (2006). Tras las líneas, sobre la lectura contemporánea. Barcelona, España: Anagrama.

Cassany, D. (2008). Prácticas letradas contemporáneas. Recuperado de http:// cmap.javeriana.edu.co/servlet/SBRe 
adResourceServlet?rid=1K249FCXL$\underline{\text { 2B7097T-2P6 }}$

De Bono, E. (2004). El pensamiento creativo, el poder del pensamiento lateral para la creación de nuevas ideas. Barcelona, España: Paidós.

De la Torre, Y. G., \& Mora, J. J. (2021). Lectura, escritura, matemáticas y habilidades digitales en la escuela. Hacia la necesidad de alfabetizaciones académicas continuas. Actualidades Investigativas en Educación, 21(1), $1-22$

Gamboa, A. (2016). Calidad de la educación superior: pretensiones y realidades institucionales. Bogotá, Colombia: Ecoe Ediciones.

Henao, O. \& Ramírez D. (2007). Estrategias de exploración y construcción de significado utilizadas por estudiantes de educación básica durante la lectura de textos en formato hipermedial. Innovar, 17(30): 47-58.

Henao, O. \& Ramírez D. (2012). ¿Cómo enseñar el área de lenguaje con un enfoque constructivista, utilizando en el aula un computador, un videoproyector e internet? Revista Educación y Pedagogía, 24 (62): 219- 230.

Hernández-Suárez, César Augusto, Prada-Núñez, Raúl, \& GamboaSuárez, Audin Aloiso. (2017). Conocimiento y uso del lenguaje matemático en la formación inicial de docentes en matemáticas. Revista de Investigación, Desarrollo e Innovación, 7(2), 287-299. https:// dx.doi.org/10.19053/20278306. v7.n2.2017.6071
Jurado, F \& Bustamante, G. (2015). Por una diversidad de textos en el aula de clase. Algunos criterios para la selección de textos escolares. En Lomas, C. \& Jurado, F. (Eds), Los libros de texto: ¿tradición o innovación? (pp. 71- 96). Bogotá, Colombia: Editorial Magisterio.

Levratto, V. \& Aparici, R. (2014). Arquitectura. Modalidades de lectura en la web. España: UNED.

Lomas, C. (2015). Libros de texto y enseñanza del lenguaje: entre la realidad y el deseo. En Lomas, C. \& Jurado, F. (Eds), Los libros de texto: ¿tradición o innovación? (pp. 31- 56). Bogotá, Colombia: Editorial Magisterio.

Martínez, M. (2006). Ciencia y arte en la investigación cualitativa. México: Trillas.

Mejía, M, (2004) Expedición pedagógica nacional. Reflexionando sobre la sistematización. En Encuentro de Sistematización de Experiencias. Recuperado de http://biblioteca.hegoa. ehu.es/system/ebooks/20190/original/ Aportes57.pdf?1468231023.

Ministerio de Educación Nacional. (2006). Estándares básicos por competencias. Recuperado de: http://redes. colombiaaprende.edu.co/ntg/men/ archivos/Referentes Calidad/ Estandares basicos de competencias/ Estandares Basicos Competencia.

Murcia, A. \& Jaramillo, J. (2008). Investigación cualitativa "La complementariedad", una guía para abordar estudios sociales. Armenia, Colombia: Kinesis. 
Moreira, H. N., \& Moreira, G. L. (2020). literacidad visual y crítica en ele para fines específicos: lectura de anuncios publicitarios como práctica social y política. Trabalhos em Linguística Aplicada, 59(2), 1455-1480.

Monteiro, A., \& Leite, C. (2020). Alfabetizaciones digitales en la educación superior: Habilidades, usos, oportunidades y obstáculos para la transformación digital. Revista De Educación a Distancia (RED), 21(65). https://doi.org/10.6018/ red. 438721

Robinson, K. (2011). El elemento. Recuperado de http://craig.com.ar/biblioteca/El\%20 elemento $\% 20-\% 20$ Descubrir $\% 20$ tu\%20pasi $\%$ F3n $\% 2010 \% 20$ cambia $\% 20$ todo $\% 20-\% 20$ Ken $\% 20$ Robinson.pdf

Rivas, N. L. (2020). La competencia lecto-crítica en entornos digitales: una visión holística para L2/LE. Contextos educativos: Revista de educación, (25), 71-89.

Solé, I. (1987). Las posibilidades de un modelo teórico para la enseñanza de la comprensión lectora. Infancia y aprendizaje, 10(39-40), 1-13.

Zubiría, J. (2013). ¿Cómo diseñar un currículo por competencias? Fundamentos, lineamientos y estrategias. Bogotá, Colombia: Cooperativa editorial Magisterio. 